\title{
Darf Ihr Herzpatient auf die Berge?
}

Heute kann jeder, ob gesund oder krank, mit der Seilbahn hohe Berge erreichen und dort den unterschiedlichsten Freizeitaktivitäten nachgehen. Was darf der Arzt seinen Patienten mit kardialen Krankheiten erlauben?

— Die Autoren haben eine umfassende Literaturrecherche zum Thema „Herzkrankheiten und Höhe“ ab dem Jahr 1976 bis heute gemacht. Vier Publikationen berichten über Belastungsuntersuchungen bei Patienten mit stabiler koronarer Herzkrankheit und nach Myokardinfarkt in geringen (bis $1500 \mathrm{~m}$ ), mittleren (1500 bis $2500 \mathrm{~m}$ ) und großen (über $2500 \mathrm{~m}$ ) Höhen. Es kann in der Höhe zwar schon bei geringeren Belastungen zu Angina und ischämischen EKG-Veränderungen als in der Ebene kommen, aber bei vergleichbarem myokardialem Sauerstoffverbrauch (gemessen am FrequenzDruck-Produkt). Daraus leiten die Autoren ab, dass der Aufenthalt in mittleren und großen Höhen auch für KHK-
Patienten sicher ist, allerdings sollten sie dort nur 80-90\% der auf der Wohnebene maximal möglichen Leistung erbringen.

Im Vergleich mit den üblichen, vorsichtigen Ratschlägen sind weitere Empfehlungen großzügiger: Patienten mit instabiler Angina, ventrikulären und supraventrikulären Extrasystolen, nicht kompensierter Herzinsuffizienz, Herzinfarkt in den letzten zwei Wochen, Revaskularisierung oder Thoraxoperationen in den letzten drei Wochen dürfen auf die Berge, sollten sich aber oberhalb geringer Höhen nicht belasten. Patienten mit schwerer Herzinsuffizienz oder Angina sollten sich nicht auf Höhen über $2500 \mathrm{~m}$ begeben. Unverändert wird empfohlen: Bei allen Personen jenseits des 40. Lebensjahres und Patienten mit Herzerkrankungen sollte vor einem Höhenaufenthalt ein Belastungstest durchgeführt werden.

KOMMENTAR: Wenn die Aussagen der Publikation in dieser Kürze zitiert wer- den, regt sich Widerspruch. Doch fordern die Autoren selbstverständlich vor der Auffahrt zunächst die konsequente Behandlung von Herzerkrankungen wie Angina pectoris, Herzinsuffizienz und Herzrhythmusstörungen. Wenn jedoch eine optimale Therapie nicht zu Normalisierung und Beschwerdefreiheit führt, ist die Gefährdung der Patienten in der Höhe nicht größer als auf ebener Erde - abgesehen von evtl. Evakuierungsproblemen im Notfall.

In der Regel antizipieren vernünftige Patienten mit chronischen Herzerkrankungen sehr gut, was sie sich - in der Ebene und auf den Bergen - zutrauen und was sie leisten können. Die eigentliche Gefährdung stammt aus einer überhöhten Leistungsanforderung bis ins hohe Alter und der Überschätzung der Leistungsfähigkeit. Da will der über 50-Jährige, evtl. nach einem Herzinfarkt, es sich und allen noch einmal zeigen, dass er wie als 20-Jähriger immer noch die gleichen Gipfel erreichen kann. Solche Patienten aber fragen ohnehin nicht ihren Arzt, geschweige denn befolgen sie seinen Rat.

H. HolzGREVE =

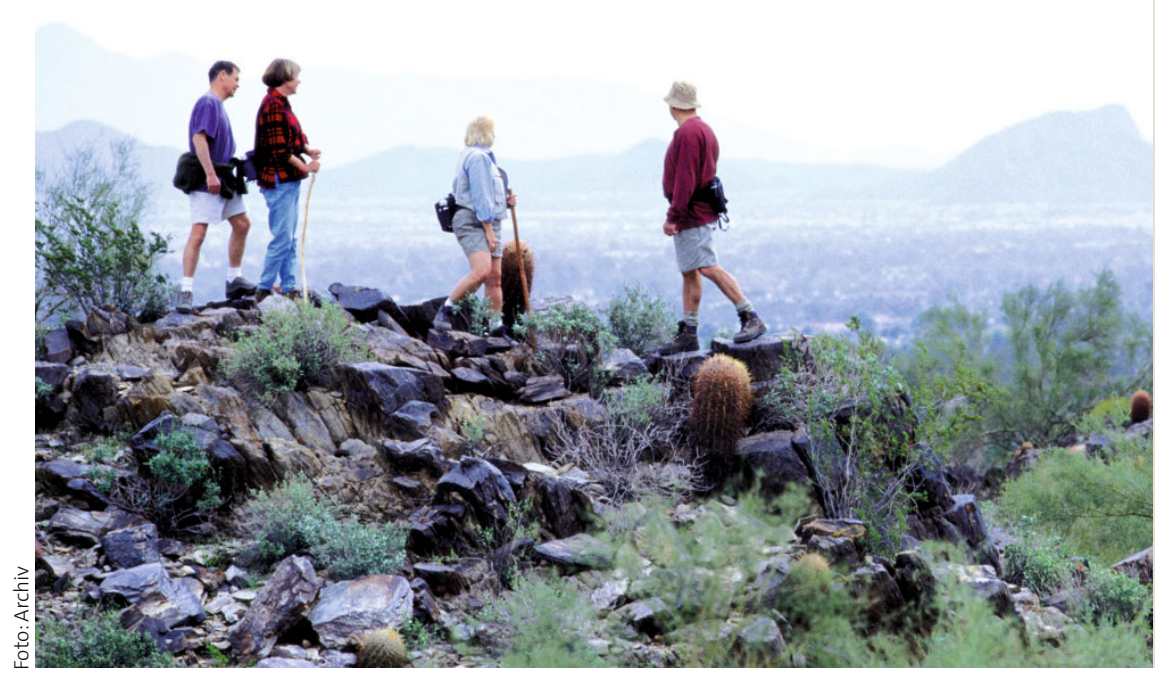

Gefährlich wird eine Bergwanderung für Herzpatienten nur dann, wenn sie ihre eigene Leistungsfähigkeit überschätzen.
J. P. Higgins et al.

Altitude and the heart: is going high safe for your cardiac patient? Am. Heart J. 159 (2010) $25-32$ 